\title{
Flare lines in Hinode EIS spectra
}

\author{
G. Del Zanna \\ 1 University College London, MSSL Holmbury St. Mary Dorking RH5 6NT, UK \\ 2 DAMTP, Centre for Mathematical Sciences, Wilberforce road Cambridge CB3 0WA, UK \\ e-mail: GDelZanna@spd.aas.org \\ Received 8 November 2007 / Accepted 31 December 2007

\section{ABSTRACT}

\begin{abstract}
We aim to identify the brightest hot emission lines in Hinode EIS spectra, and suggest the best ones to be used in flare observations. The EIS database was searched in order to find suitable datasets to identify the prominent lines. A B2-class flare spectrum was found. Most previous identifications are confirmed, however some are revised by noting the presence of blending. A new Fe XVII self-blend is identified. A recommended list of emission lines formed at high temperatures is provided. The brightest lines are the Ca XVII $192.85 \AA$ and the Fe XXIV $192 \AA$, however they are blended. The best unblended lines are the Ca XV $200.99 \AA$, Fe XVII $269.42 \AA$ and Fe XXIII $263.76 \AA$ lines.
\end{abstract}

Key words. Sun: flares - line: identification - Sun: corona

\section{Introduction}

One of the goals of the Hinode mission is to determine the mechanisms responsible for transient phenomena, such as flares. Since October 2006, the Hinode EUV Imaging Spectrometer (EIS, see Culhane et al. 2007) has observed the solar corona

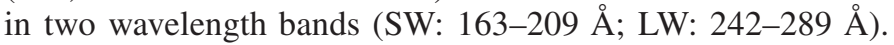
These bands were chosen to cover a wide temperature range, in particular the higher temperatures found in active regions and flares. The average EIS telemetry rate is about $50 \mathrm{kbits} / \mathrm{s}$, which is normally just enough to allow a few spectral lines to be downloaded from every exposure. This restriction is one of the reasons why much effort has been devoted to identifying the best spectral lines to be used for diagnostics. Del Zanna \& Mason (2005) presented a complete list of the strongest EIS lines expected to be observed for the quiet Sun, active region and flares. Young et al. (2007a) presented a preliminary discussion of early EIS spectra of the quiet Sun and active regions, while Young et al. (2007b) confirmed the presence of transition region lines, prominent in the legs of active region loops.

This paper provides a preliminary guide to the most prominent lines formed at high temperatures. One key aspect is the consideration of total observed counts in the lines, given that the effective areas in the two EIS channels are highly peaked, and only lines close to the peak have large count rates. The NRL/ATM slitless spectrograph (S082A) onboard Skylab produced a number of excellent spectra of flares in the 171-630 $\AA$ range, which allowed a number of identifications to be made (cf. Sandlin et al. 1976; Dere 1978). The advantage of EIS is the ability to clearly separate the spatial and spectral components of the emission. One disadvantage is the time needed to scan an area of the Sun with the slit. Another one is the lack of co-spatiality between the two channels.

The S082A sensitivity decreased significantly at shorter wavelengths, so the EIS SW wavelengths are still relatively unexplored in terms of weaker lines. Overall, the EIS bands are

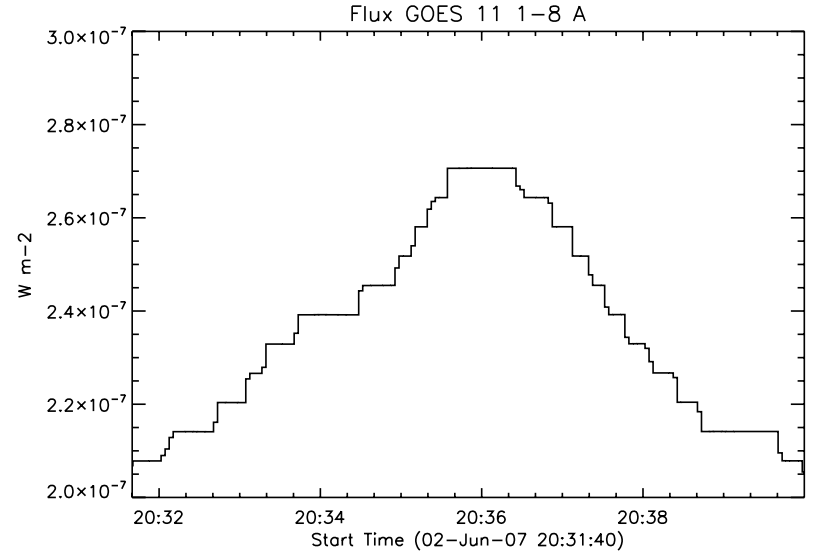

Fig. 1. X-ray flux light curve from GOES (1-8 Å) showing the B2-class flare observed by EIS.

still largely unexplored. The most extensive dataset before EIS was from the SOHO CDS grazing incidence spectrometer (GIS) (Del Zanna 1999). This Letter is part of an on-going work to calculate, benchmark, and provide reliable atomic data and line identifications for the XUV.

There is particular interest in studying lines that are formed above a few MK, mainly because they can provide information about the nature of the heating in the solar corona (see, e.g. Patsourakos \& Klimchuk 2006). As disussed e.g. in Del Zanna \& Mason (2003), lines formed between 2 and 4 MK are normally present in the "hot X-ray" loops seen in the cores of active regions. Lines formed at higher temperatures are normally shortlived and only observed during flares. Above $10 \mathrm{MK}$, prominent lines in the EIS bands are from Fe XXIII and Fe XXIV. Atomic data for these ions are discussed in Del Zanna et al. (2005) and Del Zanna (2006). In the 4-10 MK range, the most important ions for EIS are CaXVII and Fe XVII. Fe XVII produces many $3 s-3 p$ and $3 p-3 d$ transitions that fall into the EIS 

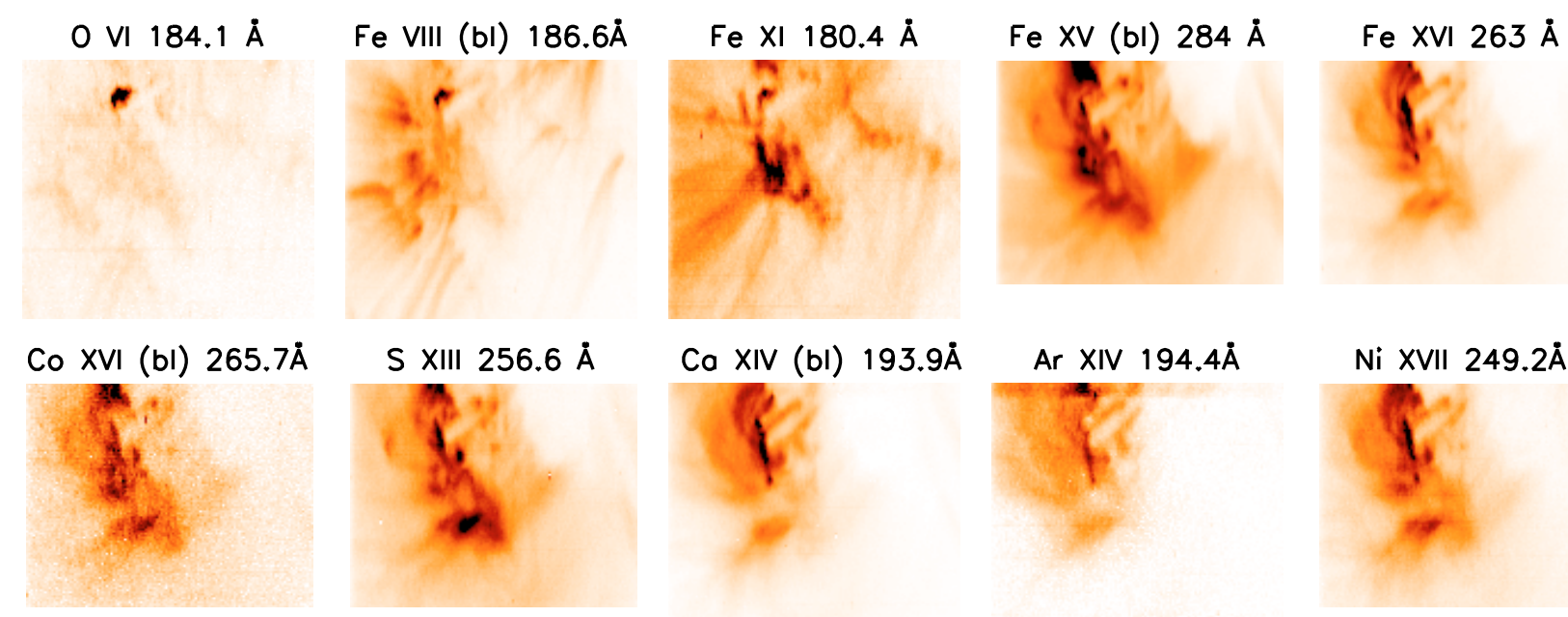

Ni XVII $249.2 \AA$
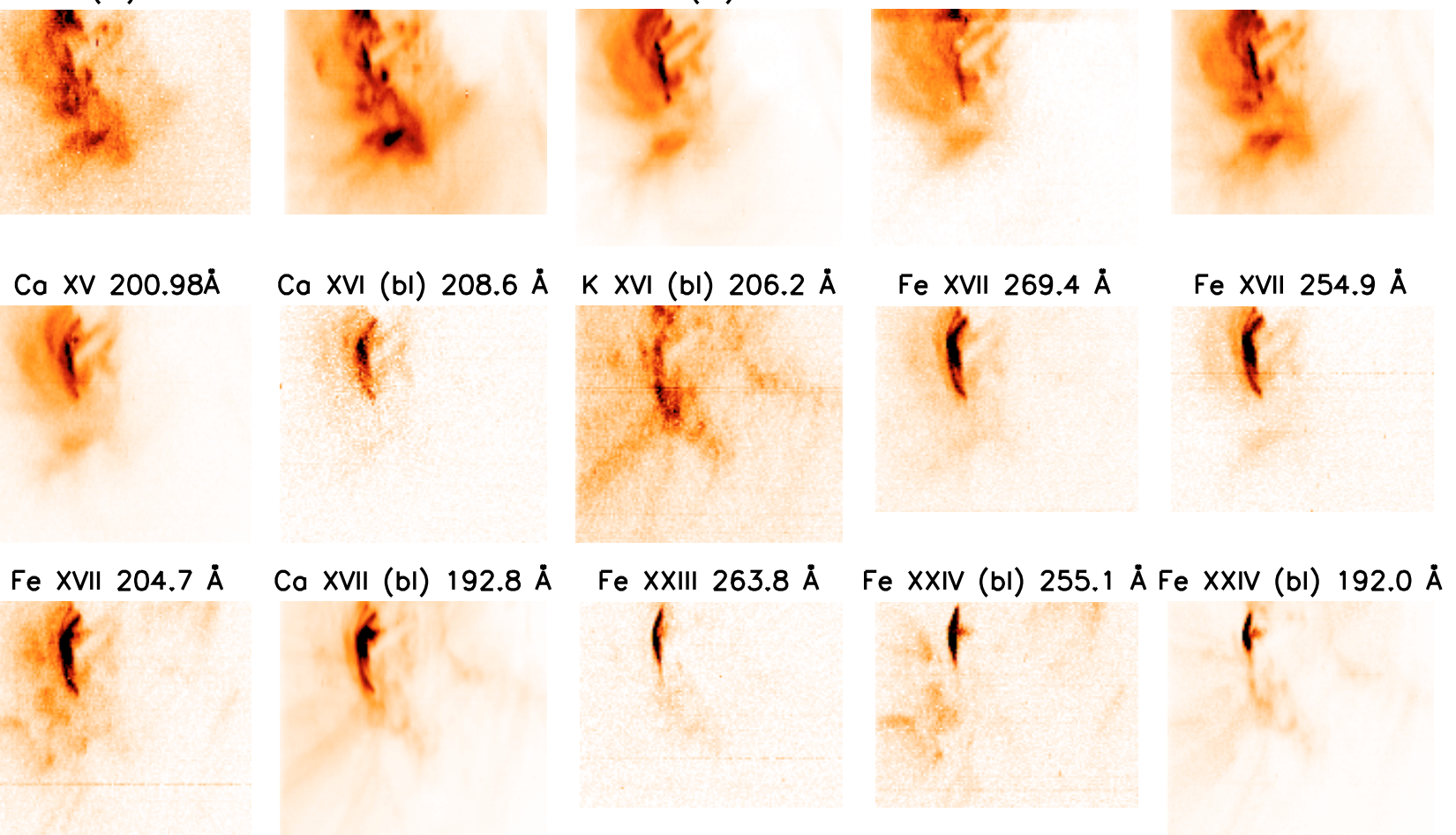

Fig. 2. Monochromatic images (negative) of a selection of EIS lines, starting with a few lines from the transition region (O VI) up to Fe XXIV, the hottest ion observed.

bands, with contradicting identifications found in the literature. Among the levels which produce EUV lines, the $3 \mathrm{p}^{1} \mathrm{~S}_{0}$ (identified by Dere 1978 with the lines at 204.6 and 254.9 $\AA$ in Skylab spectra) has an observed energy largely at odds with the best theoretical predictions (Ishikawa, priv. comm.), unlike the other levels within the same configuration. The new R-matrix atomic calculations recently published by Loch et al. (2006) predict Fe XVII line intensities that are largely different (factors of 2-3) from previous estimates for some lines, and it is therefore important to benchmark these latest calculations and reassess previous identifications.

\section{Method}

The entire set of over 46000 "raster" observations performed by EIS with the $1^{\prime \prime}$ and $2^{\prime \prime}$ slits during the first 10 months of operation has been searched for suitable datasets. The main criterion was that at least the Fe XXIV 192, and the Fe XVII 204.6 and $254.9 \AA$ wavelengths were observed. A large fraction of the spectra did not contain these wavelengths. A large number of rasters were over very small spatial regions and were discarded because of the difficulty in estimating the spatial offsets between the two channels. Almost all of the remaining observations did not have significant count rates in the "hot" lines. Some datasets were found to have strong (high-density) transition region emission, superimposed on "hot" flare emission. In these cases, it becomes impossible to estimate the formation temperature of unidentified spectral lines, and assess blending. In one case it was found that weak flaring emission was so short lived that was only observed in one of the channels.

The EIS spectral resolution allows relative measurements of line shifts of a few $\mathrm{km} \mathrm{s}^{-1}$. However, a strong (about $70 \mathrm{~km} \mathrm{~s}^{-1}$ ), non-reproduceable variation of the wavelength scale along the orbit is present, and needs to be carefully corrected for. Here, a simple correction based on the orbital variation of some of the strongest lines in an area not affected by the flare was used. The EIS wavelength calibration is also not trivial, mainly because of the lack of reference wavelengths, and of standards. Improving wavelengths is part of a long-term on-going benchmark work (cf. Del Zanna et al. 2004, and following papers). Here, the standard EIS wavelength calibration was adopted, which provides wavelengths accurate to within 10-20 m $\AA$. The EIS radiometric end-to-end calibration done on the ground at RAL has been adopted here. Work is on-going to validate it, as done for CDS (Del Zanna et al. 2001). The data have been processed with user-written procedures. The atomic data used in this paper are mostly from the version 5.2 of the CHIANTI atomic package (Landi et al. 2006). The identifications presented here are based on morphology, wavelengths and comparisons with a number of 


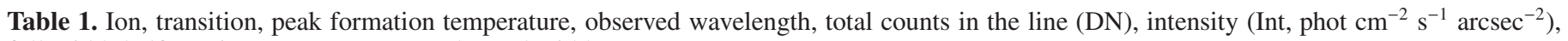
full-width-half-maximum $(F W H M)$, non-thermal width $w$.

\begin{tabular}{|c|c|c|c|c|c|c|c|}
\hline 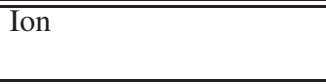 & Transition & $\begin{array}{l}\mathrm{Log} \\
T[\mathrm{~K}] \\
\end{array}$ & $\begin{array}{l}\lambda \\
(\AA)\end{array}$ & $\overline{\mathrm{DN}}$ & Int & $\begin{array}{l}F W H M \\
(\AA)\end{array}$ & $\begin{array}{l}w \\
\left(\mathrm{~km} \mathrm{~s}^{-1}\right)\end{array}$ \\
\hline \multicolumn{8}{|c|}{ A selection of lines with increasing formation temperature (area of Fe XVII peak emission) } \\
\hline $\mathrm{O}$ VI & $1 \mathrm{~s}^{2} 2 \mathrm{p}^{2} \mathrm{P}_{3 / 2}-1 \mathrm{~s}^{2} 3 \mathrm{~s}^{2} \mathrm{~S}_{1 / 2}$ & $5.5^{1}$ & 184.11 & 426 & 102.8 & 0.120 & 107 \\
\hline Fe VIII (bl Ca XIV) & $3 p^{6} 3 d^{2} D_{3 / 2}-3 p^{5} 3 d^{2}{ }^{2} F_{5 / 2}$ & 5.8 & 186.61 & 2230 & 292.2 & 0.104 & 88 \\
\hline $\mathrm{Fe} \mathrm{XI}(\mathrm{bl} \mathrm{Fe} \mathrm{X} \mathrm{)}$ & $3 s^{2} 3 p^{4}{ }^{3} P_{2}-3 s^{2} 3 p^{3} 3 d^{3} D_{3}$ & 6.1 & 180.40 & 807 & 655.4 & 0.078 & 59 \\
\hline Fe XII & $3 s^{2} 3 p^{3}{ }^{4} S_{3 / 2}-3 s^{2} 3 p^{2} 3 d^{4} P_{3 / 2}$ & 6.2 & 193.51 & 11572 & 609.3 & 0.075 & 51 \\
\hline Fe XV (bl) & $3 \mathrm{~s}^{2}{ }^{1} \mathrm{~S}_{0}-3 \mathrm{~s} 3 \mathrm{p}^{1} \mathrm{P}_{1}$ & 6.3 & 284.16 & 13174 & 7012.1 & 0.108 & 52 \\
\hline Fe XVI (bl ?) & $3 p^{2} P_{3 / 2}-3 d^{2} D_{5 / 2}$ & 6.4 & 262.98 & 7679 & 1786.6 & 0.098 & 46 \\
\hline Co XVI (bl S XIII) & $3 \mathrm{~s}^{2}{ }^{1} \mathrm{~S}_{0}-3 \mathrm{~s} 3 \mathrm{p}^{1} \mathrm{P}_{1}$ & 6.4 & 265.74 & 177 & 36.3 & 0.084 & 31 \\
\hline S XIII & $2 \mathrm{~s}^{2}{ }^{1} \mathrm{~S}_{0}-2 \mathrm{~s} 2 \mathrm{p}^{1} \mathrm{P}_{1}$ & 6.4 & 256.68 & 2140 & 727.6 & 0.099 & 43 \\
\hline Ca XIV (bl Ar XIV) & $2 s^{2} 2 p^{3}{ }^{4} S_{3 / 2}-2 s 2 p^{4}{ }^{4} P_{1 / 2}$ & 6.5 & 183.46 & 392 & 114.7 & 0.120 & 102 \\
\hline Ar XIV (bl u X) & $2 \mathrm{~s}^{2} 2 \mathrm{p}^{2} \mathrm{P}_{3 / 2}-2 \mathrm{~s} 2 \mathrm{p}^{2}{ }^{2} \mathrm{P}_{1 / 2}$ & 6.5 & 191.41 & 922 & 57.9 & 0.106 & 81 \\
\hline Ca XIV (bl) & $2 s^{2} 2 p^{3}{ }^{4} S_{3 / 2}-2 s 2 p^{4}{ }^{4} P_{5 / 2}$ & 6.5 & 193.87 & 4869 & 250.8 & 0.079 & 46 \\
\hline Ar XIV (bl ?) & $2 s^{2} 2 p^{2} P_{1 / 2}-2 s 2 p^{2} S_{1 / 2}$ & 6.5 & 194.41 & 1539 & 77.2 & 0.107 & 80 \\
\hline Ni XVII & $3 \mathrm{~s}^{2}{ }^{1} \mathrm{~S}_{0}-3 \mathrm{~s} 3 \mathrm{p}^{1} \mathrm{P}_{1}$ & 6.5 & 249.18 & 1434 & 909.5 & 0.090 & 41 \\
\hline \multicolumn{8}{|c|}{ Flare lines (area of Fe XVII peak emission) } \\
\hline $\mathrm{Ca} \mathrm{XV}$ & $2 \mathrm{~s}^{2} 2 \mathrm{p}^{2}{ }^{3} \mathrm{P}_{0}-2 \mathrm{~s} 2 \mathrm{p}^{3}{ }^{3} \mathrm{D}_{1}$ & 6.6 & 200.99 & 2372 & 290.4 & 0.093 & 59 \\
\hline Ca XVI (bl Ca XV) & $2 \mathrm{~s}^{2} 2 \mathrm{p}^{2} \mathrm{P}_{1 / 2}-2 \mathrm{~s} 2 \mathrm{p}^{2}{ }^{2} \mathrm{D}_{3 / 2}$ & 6.6 & 208.60 & 397 & 370.9 & 0.114 & 78 \\
\hline K XVI (bl) & $2 \mathrm{~s}^{2}{ }^{1} \mathrm{~S}_{0}-2 \mathrm{~s} 2 \mathrm{p}^{1} \mathrm{P}_{1}$ & 6.6 & 206.25 & 146 & 89.8 & 0.074 & 23 \\
\hline Fe XVII & $2 p^{5} 3 s^{1} P_{1}-2 p^{5} 3 p^{1} S_{0}$ & 6.6 & 204.65 & 780 & 345.1 & 0.112 & 82 \\
\hline Fe XVII & $2 p^{5} 3 s^{3} P_{1}-2 p^{5} 3 p^{1} S_{0}$ & 6.6 & 254.88 & 501 & 193.7 & 0.112 & 58 \\
\hline Fe XVII & $2 p^{5} 3 p^{3} D_{2}-2 p^{5} 3 d^{3} F_{3}$ & 6.6 & 269.42 & 678 & 126.1 & 0.117 & 58 \\
\hline Fe XVII (sbl) & $3 \mathrm{p}^{1} \mathrm{D}_{2},{ }^{3} \mathrm{P}_{2}-3 \mathrm{~d}{ }^{1} \mathrm{~F}_{3},{ }^{3} \mathrm{D}_{3}$ & 6.6 & 280.15 & 627 & 213.9 & 0.154 & 85 \\
\hline Ca XVII (bl Fe XI, O V) & $2 \mathrm{~s}^{2}{ }^{1} \mathrm{~S}_{0}-2 \mathrm{~s} 2 \mathrm{p}^{1} \mathrm{P}_{1}$ & 6.7 & 192.85 & 23979 & 1323.0 & 0.121 & 93 \\
\hline Fe XXIII (bl Ar XV ?) & $2 \mathrm{~s}^{2}{ }^{1} \mathrm{~S}_{0}-2 \mathrm{~s} 2 \mathrm{p}^{3} \mathrm{P}_{1}$ & 7.1 & 263.76 & 353 & 79.0 & 0.120 & 31 \\
\hline Fe XXIV (bl Fe XI) & $1 s^{2} 2 s^{2} S_{1 / 2}-1 s^{2} 2 p^{2} P_{3 / 2}$ & 7.1 & 192.02 & 4683 & 277.1 & 0.127 & 109 \\
\hline Fe XXIV (bl S X) & $1 s^{2} 2 s^{2} \mathrm{~S}_{1 / 2}-1 \mathrm{~s}^{2} 2 \mathrm{p}^{2} \mathrm{P}_{1 / 2}$ & 7.1 & 255.11 & 316 & 120.4 & 0.149 & 64 \\
\hline \multicolumn{8}{|c|}{ Flare lines (area of Fe XXIV peak emission) } \\
\hline Fe XXIII (bl Ar XV ?) & & 7.1 & 263.77 & 350 & 78.3 & 0.124 & 38 \\
\hline Fe XXIV (bl) & & 7.1 & 192.03 & 10823 & 639.8 & 0.120 & 102 \\
\hline Fe XXIV (bl) & & 6.1 & 255.12 & 292 & 111.0 & 0.128 & 76 \\
\hline
\end{tabular}

different EIS spectra (quiet Sun, on-disk and off-limb, active regions). Whenever available, ratios of lines emitted from the same ion have been checked, to assess identification and the importance of blending.

\section{Results and discussion}

Only one suitable dataset has been found so far. It is a full spectral atlas over AR 10960, scanned (from West to East) on June 2nd 2007, with the $1^{\prime \prime}$ (and exposures of $25 \mathrm{~s}$ ) between 19:56:12 and 20:52:52 UT. AR 10960 produced two M-class flares earlier on that day around 5:30 and 10:30 UT and was relatively active. A B2-class flare occurred between 20:32 and 20:38 (cf. Fig. 1).

Fe XXIV emission in the EIS SW channel was observed in the interval 20:35:10-20:36:56 UT. Peak emission was observed in the exposure taken at 20:36:03 UT. The approximate east-west offset of the LW channel is $2^{\prime \prime}$, and therefore the same region was recorded in the LW spectra after $1 \mathrm{~min}$. Discrepancies of more than a factor of 2 between the Fe XXIV lines observed in the two channels have been observed. These are most likely caused by real changes of the Fe XXIV intensities (the GOES light curve indicates that this was the time of maximum variation, its small value is probably due to the spatial and temperature averaging of the GOES instrument). This clearly points out the difficulty in using diagnostics that involve lines in both EIS channels.

The spectral line profiles in this observation are nearly Gaussian, even in the flaring region (an exact shape is difficult to assess, given the the fact that lines are dispersed over only a few pixels). Intensities of a few hundreds of lines during the scan have been obtained. Figure 2 shows the resulting monochromatic images of a selection of lines. The images from the LW channel have been shifted by $2^{\prime \prime}, 17^{\prime \prime}$ in the E-W, N-S direction to approximately correct for the offset between the two channels.

Notice a strong transition-region (TR) emission (O VI), which fortunately is not superimposed on the hot emission (Fe XVII, Fe XXIV). Another fortunate occurrence is that lines at different temperatures present a clearly different morphology. Fe XXIV emission is spatially confined to a loop-like structure, while lines from Ca XVI, K XVI, Fe XVII, Ca XVII clearly show two twisted and interconnecting loops, which perhaps indicate a signature of a reconnection event taking place in the corona. The morphology of all the hundreds of lines recorded by EIS has been studied, to identify their formation temperature.

Three averaged spectra were obtained, one from the area of peak Fe XVII emission (a few spectral windows are shown in Fig. 3), one from the area of peak Fe XXIV emission, and one from the strong TR emission. Table 1 shows a summary of the line identifications. Notice the large differences between observed counts and calibrated units, due to the strongly peaked effective areas. There are regions where lines are very broad, most notably where hot lines are present, and in particular where the TR emission is located. A rough value of the non-thermal broadening, calculated using the instrumental widths measured during the ground calibration, is added to Table 1.

The hottest lines observed are from Fe XXIII, Fe XXIV. The doublet $1 \mathrm{~s}^{2} 2 \mathrm{~s}^{2} \mathrm{~S}_{1 / 2}-1 \mathrm{~s}^{2} 2 \mathrm{p}^{2} \mathrm{P}_{3 / 2,1 / 2}$ is observed at 192.03 and $255.11 \AA$ respectively. The $255.11 \AA$ line is contaminated with 

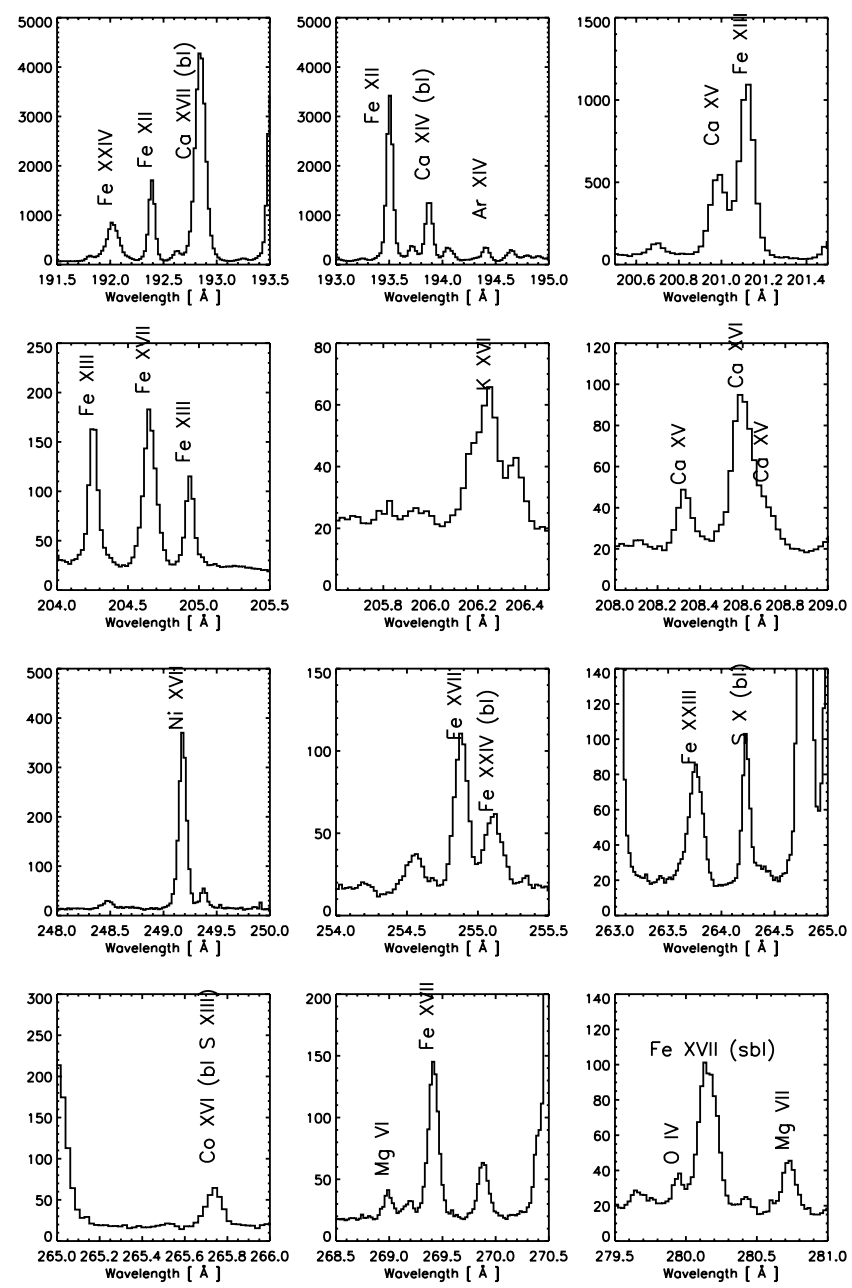

Fig. 3. A portion of the averaged spectrum (units are counts) over an area where Fe XVII was brightest.

unidentified $1 \mathrm{MK}$ emission (at least from a relatively weak S X $255.06 \AA$ ), and the same is true for the $192.03 \AA$ line (with a previously unidentified Fe XI line). However, even for a B-class flare such as this one, the dominant emission in these spectral lines appears to be due to Fe XXIV. The Fe XXIII intercombination $2 \mathrm{~s}^{2}{ }^{1} \mathrm{~S}_{0}-2 \mathrm{~s} 2 \mathrm{p}{ }^{3} \mathrm{P}_{1} 263.76 \AA$ line appears to be unblended and is therefore one of the best high-temperature lines observed by EIS.

Ca XVII $198.9 \AA$ is significantly blended with at least 4 transitions, from $\mathrm{OV}$ and Fe XI. The stronger of the $\mathrm{OV} 2 \mathrm{p}^{3} \mathrm{P}_{\mathrm{J}}-$ $3 \mathrm{~d}{ }^{3} \mathrm{D}_{\mathrm{J}}$ transitions is at $192.90 \AA$ and can be isolated from the Fe XI-Ca XVII-O V feature at $192.8 \AA$ only in some circumstances. Whenever (as here) significant non-thermal broadening is present, the line becomes blended, and it becomes non-trivial to deblend the $\mathrm{O}$ V contribution. Fe XI is a complex ion, and some identifications found in the literature of even the strongest lines observed by EIS are incorrect. Work is in progress to calculate reliable atomic data and confirm identifications. It is anticipated here that the $3 \mathrm{~s}^{2} 3 \mathrm{p}^{3}\left({ }^{2} \mathrm{D}\right) 3 \mathrm{~d}^{3} \mathrm{P}_{2}$ level decays to the ground configuration $3 \mathrm{~s}^{2} 3 \mathrm{p}^{4}{ }^{3} \mathrm{P}_{2,1}$ levels to form the $188.22 \AA 192.81 \AA$ lines. The $188.22 \AA$ line appears largely unblended, and therefore can be used (if observed) to deblend the $192.81 \AA$ contribution.

The identification of the FeXVII branching ratio 204.65, $254.88 \AA$ is confirmed, mostly based on the fact that no other bright candidate lines with the correct wavelength separation have been found. However, the $254.88 \AA$ appears to be weaker by more than 50\%, compared to predictions (the Loch et al. 2006, data predict an equal intensity for this branching ratio). Various other discrepancies have been found, and work is on-going to assess whether they are due to problems in the atomic data or in the EIS radiometric calibration. The line observed at $280.15 \AA$ shows the same morphology as the other Fe XVII lines, and is obviously a blend. It is identified here as the Fe XVII self-blend of the $3 p{ }^{1} D_{2},-3 d{ }^{1} F_{3}$ and $3 p{ }^{3} P_{2}-3 d^{3} D_{3}$ transitions. This is based on a good agreement between observed and predicted intensity (using the Loch et al. 2006 data) relative to the $269.42 \AA$ line. EIS is well-suited to study $2-4 \mathrm{MK}$ emission, given the large number of strong lines: the resonance S XIII $256.68 \AA$, Ni XVII 249.18 ̊, Fe XV 284.15 ̊ (though blended with multiple transitions) lines, together with the Fe XVI $262.98 \AA$ line.

\section{Conclusions}

A list of prominent EIS lines to be used for studies of hightemperature plasma is provided, based on the rare occurrence of a full spectrum of a flare (B2-class) recorded by EIS. Most lines, previously seen in Skylab spectra, were predicted to be observable by Del Zanna \& Mason (2005). Some "hot" lines, however, such as those from Fe XXI, Fe XXII, Mn XXIII, were not observed. This is probably due to the fact that this was a small flare. With the exception of an Fe XVII self-blend, previous identifications are confirmed, although many are revised by noting the presence of blending. Further work is on-going, to identify EIS lines and properly assess blending.

The best lines in terms of high count rates are the Fe XXIV $192.0 \AA$ and the Ca XVII 192.85, although for small flares accurate deblending of both lines should be done. The strongest (and possibly unblended) Fe XVII lines are observed at 204.65 and $269.42 \AA$. The Ca XV 200.99 $\AA$ and Fe XXIII $263.76 \AA$ are also excellent lines. Considering the lack of co-spatiality in the two EIS channels, it is suggested to select flare lines in either of the two bands. The potential of the EIS instrument to study flares is clearly demonstrated here.

Acknowledgements. Support from STFC (Advanced Fellowship and APAP network) is acknowledged. Dr. Traebert and Prof. Ishikawa are warmly thanked for fruitful discussions on the complex issue of line identifications along the Ne-like sequence. Dr. H. E. Mason and the referee are also thanked for useful comments on the manuscript. Hinode is a Japanese mission developed and launched by ISAS/JAXA, with NAOJ as domestic partner and NASA and STFC (UK) as international partners. It is operated by these agencies in co-operation with ESA and NSC (Norway).

\section{References}

Culhane, J. L., Harra, L. K., James, A. M., et al. 2007, Sol. Phys., 60 Del Zanna, G. 1999, Ph.D. Thesis, Univ. of Central Lancashire, UK Del Zanna, G. 2006, A\&A, 447, 761

Del Zanna, G., \& Mason, H. E. 2003, A\&A, 406, 1089

Del Zanna, G., \& Mason, H. E. 2005, Adv. Space Res., 36, 1503

Del Zanna, G., Bromage, B. J. I., Landi, E., \& Landini, M. 2001, A\&A, 379, 708

Del Zanna, G., Berrington, K. A., \& Mason, H. E. 2004, A\&A, 422, 731

Del Zanna, G., Chidichimo, M. C., \& Mason, H. E. 2005, A\&A, 432, 1137

Dere, K. P. 1978, ApJ, 221, 1062

Landi, E., Del Zanna, G., Young, P. R., et al. 2006, ApJS, 162, 261

Loch, S. D., Pindzola, M. S., Ballance, C. P., \& Griffin, D. C. 2006, J. Phys. B Atom. Mol. Phys., 39, 85

Patsourakos, S., \& Klimchuk, J. A. 2006, ApJ, 647, 1452

Sandlin, G. D., Brueckner, G. E., Scherrer, V. E., \& Tousey, R. 1976, ApJ, 205, L47

Young, P. R., Del Zanna, G., Mason, H. E., et al. 2007a, PASJ, 59, 857

Young, P. R., Del Zanna, G., Mason, H. E., et al. 2007b, PASJ, 59, 727 